\title{
Análisis univariante para describir y pronosticar la producción de plátano en la región de piura
}

\author{
Carrasco Choque Freddy \\ ORCID: https://orcid.org/0000-0002-4493-5567 \\ fcarrasco@unf.edu.pe \\ Universidad Nacional de Frontera \\ Sullana, Perú
}

\author{
Villegas Yarleque Mario \\ ORCID: https://orcid.org/0000-0001-5572-1372 \\ mvillegas@unf.edu.pe \\ Universidad Nacional de Frontera \\ Sullana, Perú
}

\author{
Sánchez Castro Janet Del Rocío \\ ORCID: https://orcid.org/0000-0001-9466-565X \\ rociosanchez252013@gmail.com \\ Universidad Nacional del Altiplano \\ Puno, Perú
}

Recibido (19/04/21), Aceptado (12/05/21)

\begin{abstract}
Resumen: La actividad agrícola en la región de Piura, es una actividad fundamental para su desarrollo, la implementación de pronósticos es una herramienta útil para los agentes económicos para una planificación y toma de decisiones acertadas. En el estudio interesan dos resultados, el primero identificar, estimar y validar un modelo ajustado para pronosticar la producción de plátano y el segundo realizar el pronóstico de la producción de plátano para el periodo de octubre de 2020 hasta octubre de 2022. Para concretizar los objetivos se realizó el análisis univariante con la metodología de Box y Jenkins. Los datos provienen del Banco Central de Reserva del Perú, se consideraron datos mensuales desde julio de 2000 hasta septiembre de 2020. Luego del cumplimiento de los supuestos, el mejor modelo ajustado para representar la producción del plátano y realizar pronósticos es un modelo autorregresivo integrado de promedio móvil o ARIMA . El pronóstico de la producción del plátano tiene una tendencia decreciente para los próximos años.
\end{abstract}

Palabras Clave: Pronostico, Series de tiempo, Modelos ARIMA, Producción agrícola

\section{Univariate analysis to describe and forecast banana production in the piura region}

\begin{abstract}
Agricultural activity in the Piura region is a fundamental activity for its development, the implementation of forecasts is a useful tool for economic agents to plan and make correct decisions. Two results are of interest in the study, the first to identify, estimate and validate an adjusted model to forecast banana production and the second to make the forecast of banana production for the period from October 2020 to October 2022. To specify the objectives, the univariate analysis was carried out with the Box and Jenkins methodology. The data comes from the Central Reserve Bank of Peru, monthly data from July 2000 to September 2020 were considered. Once the assumptions have been met, the best fit model to represent banana production and make forecasts is an Autoregressive Integrated Moving Average or ARIMA model. The forecast for banana production has a downward trend for the next few years.
\end{abstract}

Keywords: Forecast, Time series, ARIMA models, Agricultural production

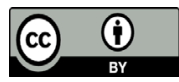




\section{Introducción}

El sector agrícola juega un papel importante en la economía de todos los países [1]. En el Perú, la agricultura se está convirtiendo en un significativo motor del crecimiento económico. En el año 2018 el producto bruto interno (PBI) del sector agropecuario creció $7.8 \%$, la tasa más alta de los últimos diez años [2].

La actividad agrícola en la región de Piura, es una actividad fundamental y fuente de riqueza permanente para sus pueblos. La agricultura ocupa el 31\% de la población económicamente activa - PEA, el desarrollo económico de la región se basa en el comercio de sus principales productos agrícolas, tanto para el mercado nacional como para el extranjero [3]. Los cultivos además de demandar mano de obra, generan ingresos y divisas, su importancia se reconoce a nivel nacional [4].

Según el Instituto Nacional de Estadística e Informática INEI [5], la agricultura representa el 8.2\% del PBI regional y aporta 5.7\% al PBI agrícola nacional. Asimismo [6], menciona que la economía regional gira en torno a la agricultura, ya sea directamente en la producción o indirectamente a través de industrias que procesan cultivos tradicionales como arroz, algodón y café, y los no tradicionales limón, mango, plátano.

Sin embargo, la operatividad, competitividad en el sector agrícola, presenta desafíos que podrían ser originados por factores climatológicos, plagas, enfermedades, de la organización, económicos y otros factores coyunturales, muchas veces con consecuencias negativas en la producción y en las ventas de sus productos [7]. Entonces, carecer de un programa de planeación y control sistemático de procesos agrícolas, implica el fracaso de cualquier esfuerzo tendiente a fomentar la sustentabilidad agrícola [8].

Por lo anterior, debemos tener en cuenta que el pronóstico posibilita las buenas decisiones futuras y proporciona estimados cuantitativos de la probabilidad de eventos futuros [9]. Lo que justifica la realización de la investigación, por su incorporación de pronósticos en la producción agrícola lo que deriva su importancia económica y social [10].

Teniendo en cuenta a [11], la técnica más común para realizar pronósticos es la metodología de Box y Jenkins, que consiste en identificar y estimar un modelo estadístico que pueda ser interpretado como generador de la información de la muestra. Por tanto, si el modelo estimado es usado para la predicción debe suponerse que las características de la serie son constantes en el tiempo, especialmente para los periodos futuros. Entonces, la predicción se efectúa sobre una base válida considerando que el modelo es estacionario y estable.

Asimismo, en [12] el modelo autorregresivo integrado de promedio móvil se puede expresar como ARIMA(p,d,q) donde los parámetros $\mathrm{p}$, d y q indican el orden de los distintos componentes del modelo, los componentes autorregresivo $\mathrm{AR}=\mathrm{p}$ indica el número de veces que se realiza la diferencia de una variable en un instante de tiempo en función de sus valores precedentes, integrado $\mathrm{I}=\mathrm{d}$ significa realizar la diferencia estacional de la serie y de media móvil $\mathrm{MA}=\mathrm{q}$ indica la realización o no de la diferencia entre un periodo y otro del término aleatorio o error del modelo. Cuando alguno de los tres parámetros es cero, es común omitir las letras correspondientes del acrónimo AR, I o MA, si el modelo es ARI$\operatorname{MA}(0,1,0)$ se puede expresar como I $(1)$, si es $\operatorname{ARIMA}(0,0,1)$ como MA(1).

Entonces, en relación a la evidencia empírica a nivel internacional de estudios de pronósticos, se puede verificar el estudio de [13] quien ajustó el modelo ARIMA para pronosticar los diferentes tipos de producción de frutas en Bangladesh. Encontró que un ARIMA $(2,1,3)$, ARIMA $(3,1,2)$ y ARIMA $(1,1,2)$ son los mejores modelos para pronosticar la producción de mango, banano y guayaba, respectivamente.

De la misma forma, para determinar la tendencia de la producción del banano de clase A y clase B en Bangladesh, en [14] se utilizó la metodología de Box y Jenkins. Los modelos identificados para realizar pronósticos de los bananos clase $\mathrm{A}$ y clase B fueron MA $(12)$ y ARIMA $(1,6,2)$ respectivamente. La producción de banano ofrece opciones adecuadas para la subsistencia y la generación de ingresos en Bangladesh.

La metodología de Box y Jenkins, también se utiliza en diferentes sectores agrícolas para pronosticar la producción agrícola. Como la investigación desarrollada por [15], quienes analizaron las perspectivas futuras de la producción del arroz en Ghana, aplicando el modelo ARIMA. El análisis revela que un ARIMA $(2,1,0)$ es el mejor modelo para pronosticar la producción de arroz. Así como en [8], quien realizó el pronóstico de la producción de caña de azúcar en México, obteniendo el mejor modelo autoregresivo integrado de medias móviles ARIMA (1,2,0).

A nivel nacional, existe evidencia empírica aplicando pronósticos en varios sectores, como la investigación realizada por [16], que usa la Metodología Box-Jenkins para el modelamiento de la serie del número de unidades de transfusión de sangre en el Hospital Regional Manuel Núñez Butrón - Puno, el mejor modelo es un SARIMA $(0,1,1)(1,1,0) 12$. También [17] identifica el mejor modelo ARIMA estacional (SARIMA) para modelar y proyectar la producción de papa en Puno, utilizando la metodología de Box-Jenkins, el mejor modelo que se encontró para la modelación y proyección es un SARIMA $(1,1,2)(1,0,1) 12$.

Dado el contexto anterior, la investigación tiene como objetivo general determinar el modelo de predicción mensual que mejor se ajusta para realizar el pronóstico de la producción del plátano en la región de Piura, de manera específica nos interesan dos resultados: i) identificar, estimar y validar el modelo identificado que mejor se ajusta para pronosticar la producción plátano y ii) realizar el pronóstico para el periodo 2020M10 hasta 2022M10 utilizando la metodología de Box y Jenkins.

\section{Desarrollo}

Piura se ubica en la costa norte de Perú cerca de la línea ecuatorial, el suelo es muy variado, con desiertos arenosos en la costa, relieves andinos en la sierra. El clima es cálido, desértico y oceánico, y corresponde a una zona subtropical. El 
recurso hídrico procede de dos fuentes hidrográficas del rio Chira y del rio Huancabamba, son ríos vivos de amplio caudal acuífero [4]. Por todas estas buenas condiciones, la región es agrícola por excelencia y es posible cultivar una variedad de productos agrícolas tropicales, subtropicales, templados y de zonas áridas.

Los cultivos de la región son permanentes debido a que la primera producción requiere en promedio de 3 a 4 años y su periodo de vida supera los 20 años, es decir se pueden cosechar durante varias temporadas, y son temporales porque son de carácter anual, el ciclo agrícola es corto, pero el valor de estos cultivos no proviene de la planta como tal, sino del volumen y calidad del producto que ella ofrece en cada cosecha. La tabla 1 muestra la producción agrícola en la región de Piura.

Tabla 1. Producción agrícola de Piura por tipo de cultivo 2019 en Tm

\begin{tabular}{crcr}
\hline Cultivos Transitorios & \multicolumn{1}{c}{ Tm } & Cultivos Permanentes & \multicolumn{1}{c}{ Tm } \\
\hline Arroz & 394793 & Café & 4731 \\
Frijol Castilla & 8510 & Limón & 172806 \\
Maíz Amarillo & 60088 & Mango & 434105 \\
Papa & 21931 & Uva & 172173 \\
Yuca & 7087 & Papaya & 6043 \\
Camote & 10881 & Plátano & 379212 \\
\hline
\end{tabular}

Fuente: Síntesis de Actividad Económica, diciembre 2019 - BCRP Sucursal Piura.

El plátano es un cultivo frutal de las regiones tropicales y subtropicales del mundo que se cultiva en aproximadamente 8,8 millones de hectáreas [7]. El plátano se cultiva por mucho tiempo en la región de Piura, a nivel nacional la producción se localiza en la zona oriental (71.5\%) y norte del país (22\%), pero el que más se exporta es la producción de la costa por su cercanía al océano Pacífico. En las regiones de Tumbes, Piura y Lambayeque, existen 3,414 hectáreas certificadas $(80 \%$ concentradas en Piura), el Valle del Chira es considerado el área con mayor potencial agrícola del departamento de Piura
[4]. El plátano es una fuente rica de calorías, contiene la mayoría de las vitaminas esenciales para la nutrición humana.

La región de Piura es el primer productor a nivel nacional de mango con 434,105 Tm y de limón con 172,806 Tm, es el segundo productor nacional de plátano con 379,212 Tm después de la región de San Martin y segundo productor de la uva con 221,223 Tm. después de la región Ica [18]. La Figura 1 , muestra la producción de las principales frutas a nivel nacional para el año 2019.

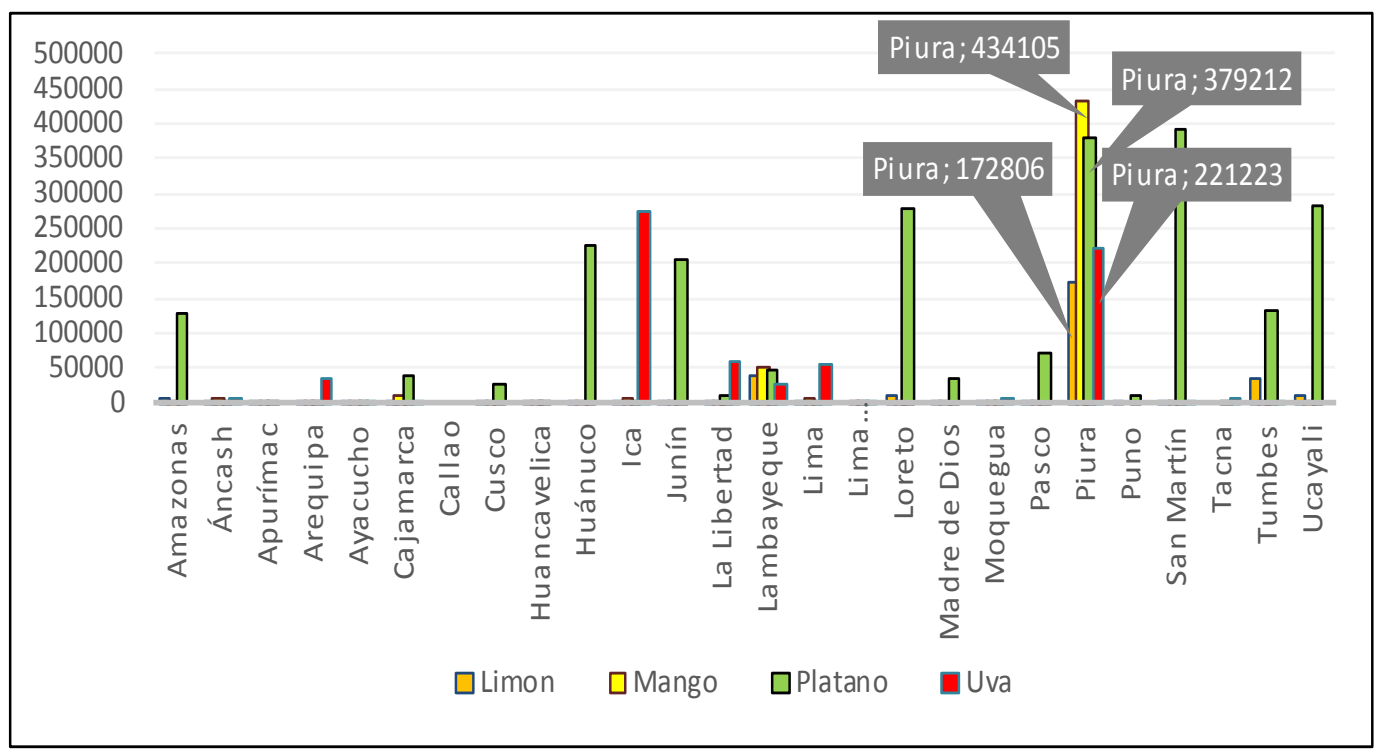

Fig. 1. Producción de frutas a nivel nacional, año 2019 en Tm

Fuente: Estadísticas del Ministerio de Agricultura y Riego. 


\section{Metodología}

A.Método.

El estudio, sigue un enfoque cuantitativo, porque se basa en la medición numérica [19]. Además, es de carácter descriptivo y correlacional. El procesamiento de datos, análisis estadístico y econométrico, se realizó utilizando el software estadístico Eviews.

\section{B.Fuente de datos.}

La serie de tiempo estudiada corresponde a la producción del plátano, en toneladas métricas, realizadas por el Banco Central de Reserva del Perú - BCRP sede Piura [20], publicada en la síntesis económica para el periodo que comprende de septiembre de 2000 hasta septiembre de 2020. El periodo de tiempo de estudio estuvo determinado por la disponibilidad de los datos, para así capturar los antecedentes de comportamiento del volumen de producción.

\section{C.Procesos autorregresivos.}

Una serie de tiempo es un conjunto de números que mide el estado de alguna actividad a lo largo del tiempo. Según [21] es el registro histórico de alguna actividad, con mediciones tomadas a intervalos igualmente espaciados con consistencia en la actividad y el método de medición.

Siguiendo con las definiciones de [12], los procesos autorregresivos son, como su nombre sugiere, regresiones sobre sí mismos. [22] realizó el trabajo original sobre procesos autorregresivos. Así, un proceso autorregresivo de orden $\mathrm{p}$, $\mathrm{AR}(\mathrm{p})$ tiene la forma

$Y_{t}=\delta+\Phi_{1} Y_{t-1}+\Phi_{2} Y_{t-2}+\Phi_{3} Y_{t-3}+\cdots+\Phi_{p} Y_{t-p}+\varepsilon_{t}$

El valor actual de la serie ${ }^{Y_{t}}$ es una combinación lineal de los valores pasados recientes de sí misma más un término de innovación ${ }^{\varepsilon_{t}}$ y es ruido blanco, que representa los errores del ajuste y otorga el carácter aleatorio al proceso.

\section{D.Procesos de media móvil.}

Estas fueron considerados por primera vez por [23] y [24]. La serie de medias móviles se puede escribir como

$Y_{t}=\delta+\varepsilon_{t}-\theta_{1} \varepsilon_{t-1}-\theta_{2} \varepsilon_{t-2}-\theta_{3} \varepsilon_{t-3}-\cdots-\theta_{q} \varepsilon_{t-q}$

A la serie se le denomina media móvil de orden y se denota como MA(q). Donde, $Y_{t}$ es la serie original y $\varepsilon_{t}$ es la serie de errores, ruido blanco con media 0 y varianza $\sigma^{2}$.

Los modelos que son una combinación de modelos AR y MA se conocen como modelos ARMA. Un modelo ARMA $(\mathrm{p}, \mathrm{q})$ se define como:

$$
\begin{aligned}
& Y_{t}=\delta+\Phi_{1} Y_{t-1}+\Phi_{2} Y_{t-2}+\cdots+\Phi_{p} Y_{t-p}+\varepsilon_{t} \\
& -\theta_{1} \varepsilon_{t-1}-\theta_{2} \varepsilon_{t-2}-\cdots-\theta_{q} \varepsilon_{t-q}
\end{aligned}
$$

Donde, $Y_{t}$ es la serie original para cada periodo $Y$, asumimos que $\varepsilon_{t}$ es independiente de $Y_{t-1}, Y_{t-2}, Y_{t-3}, \ldots, Y_{t-q}$.

Si la serie $\left\{Y_{t}\right\}$, no fuera estacionaria y tomando $d$ diferencias logramos que lo sea, tal que $W_{t}=\Delta^{d} Y_{t}$ (o también $W_{t}=Y_{t}-Y_{t-1}$ ) si es estacionaria, entonces diremos que ${ }^{\left\{Y_{\}}\right\}}$sigue un proceso autorregresivo integrado de media móvil (ARIMA) de orden $(\mathrm{p}, \mathrm{d}, \mathrm{q})$ y se denominará ARIMA $(\mathrm{p}, \mathrm{d}, \mathrm{q})$. Un proceso ARIMA $(0,1,0) \operatorname{con} W_{t}=Y_{t}-Y_{t-1}$, tendría la siguiente forma

$$
\begin{aligned}
& Y_{t}=\delta+\Phi_{1} W_{t-1}+\Phi_{2} W_{t-2}+\cdots+\Phi_{p} W_{t-p}+\varepsilon_{t} \\
& -\theta_{1} \varepsilon_{t-1}-\theta_{2} \varepsilon_{t-2}-\cdots-\theta_{q} \varepsilon_{t-q}
\end{aligned}
$$

\section{E.Metodología de Box y Jenkins.}

La metodología de Box y Jenkins [25], es el hito del enfoque moderno del análisis de series de tiempo. Dada una serie observada, el objetivo de la metodología es construir un modelo ARIMA. En particular, pasando por oportunas transformaciones preliminares de los datos, el procedimiento se centra en los procesos estacionarios. Los pasos de la metodología de Box y Jenkins, son los siguientes:

i) Análisis preliminar: crear condiciones tales que los datos disponibles puedan considerarse como la realización de un proceso estocástico estacionario. ii) Identificación de un modelo: especificar el orden (p, d, q) del modelo ARIMA, las funciones de autocorrelación empírica juegan un papel extremadamente importante. iii) Estimación del modelo: la estimación de los parámetros se puede realizar con el método de máxima verosimilitud o mínimos cuadrados ordinarios. iv) Diagnóstico: verificar si el modelo es bueno mediante pruebas de los parámetros y residuos del modelo. v) Pronostico: si el modelo cumple con los requisitos, entonces puede usarse para interpretar un fenómeno y realizar el pronóstico.

\section{F.Análisis de los residuos}

Con la finalidad de estudiar si los residuos se aproximan al comportamiento de un proceso de ruido blanco, según [26] podemos usar el estadístico $Q$ de Box-Pierce desarrollado por [27] que se basa en los coeficientes de autocorrelación de los residuos y se define como:

$Q=n \sum_{k=1}^{m} \rho_{k}^{2} \approx \chi_{m}^{2}$

Donde es el tamaño de la muestra, la longitud del rezago y es el coeficiente de autocorrelación de orden $\mathrm{k}$ de los residuos. Una variante del estadístico de Box-Pierce es el estadístico de estadístico de Ljung-Box desarrollado por [28]. 


\section{G.Prueba de normalidad de los residuos.}

Para comprobar el supuesto de normalidad de los residuos se puede utilizar el estadístico de Jarque-Bera ([29]) que es una medida de bondad de ajuste de la desviación de la normalidad, basada en la curtosis $(k)$ y la asimetría (s) muestrales. El estadístico se define como

$J B=\frac{n}{6}\left(s^{2}+\frac{(k-3)^{2}}{4}\right) \approx \chi_{(2)}^{2}$

Donde $n$ es el número de observaciones y es el número de parámetros estimados. El estadístico $J B$ tiene una distribución chi-cuadrado asintótica con 2 grados de libertad.

H.Prueba de raíz unitaria.

Siguiendo a [26], otra prueba sobre estacionariedad es conocida como la prueba de raíz unitaria, y la prueba por excelencia es la prueba de Dickey-Fuller Aumentado (DFA) de [30], esta prueba implica aumentar o adicionar valores rezagados de la variable dependiente $\Delta y_{\text {. }}$ Otro de los métodos no paramétricos para evitar la correlación serial en los términos de error, sin añadir términos de diferencia rezagados es el estadístico de Phillips-Perrón - PP de [31].

\section{Resultados}

\section{A.Modelamiento para la producción de plátano.}

El análisis preliminar de la serie (Figura 2), muestra una tendencia creciente al parecer presenta estacionariedad y quiebre estructural. Muñoz [12], menciona que el aspecto visual de la serie no siempre es una herramienta suficiente para decidir la estacionariedad.

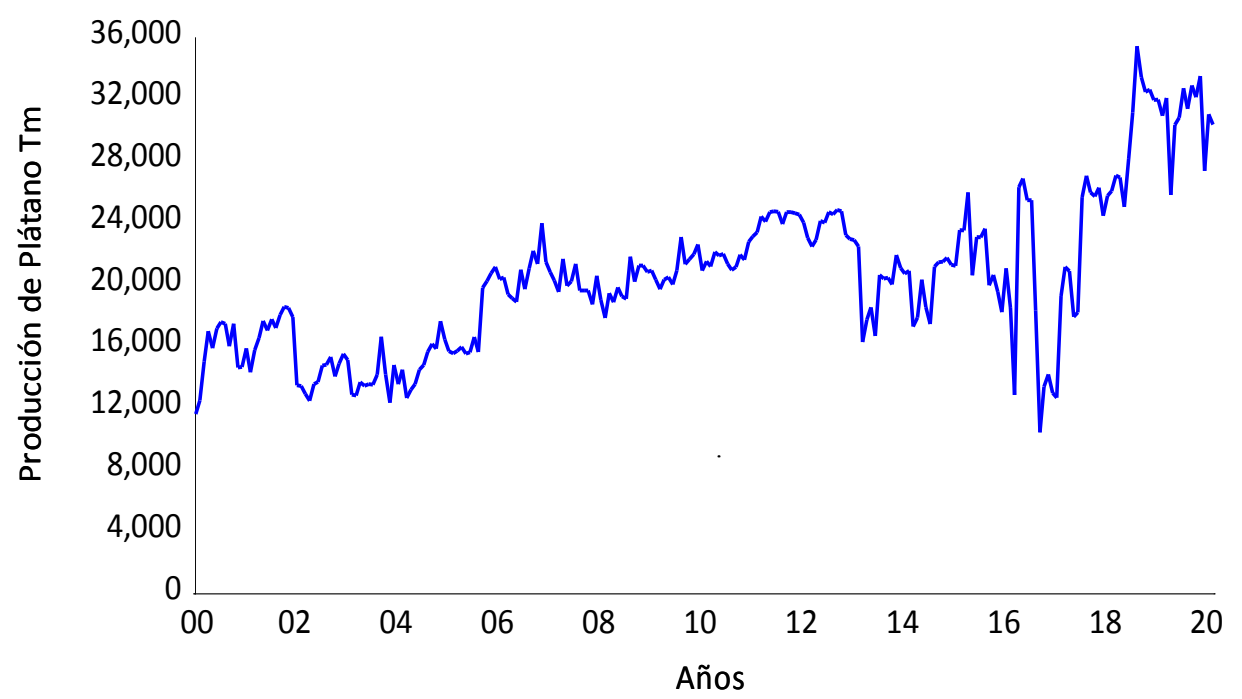

Fig. 2. Análisis gráfico de la producción de Plátano en Tm Fuente: Obtenido con base a la serie en el programa Eviews

Se comprobó la estacionariedad con la prueba estadística DFA de raíz unitaria (Tabla 2), y guiándonos de la figura 2 se realizó la prueba con intercepto y tendencia. El resultado del $t$-stadistic es $\tau=-4.801664$ y su $p$-valor $=0.0006$, entonces existe evidencia estadística para rechazar la hipótesis nula y se afirma que la serie no diferenciada es estacionaria.

Tabla 2. Análisis de raíz unitaria para detectar estacionariedad

\begin{tabular}{cccc}
\hline Augmented Dickey-Fuller test statistic & t-Statistic & Prob \\
& & -4.801664 & 0.0006 \\
\hline Test critical values: & $1 \%$ level & -3.996592 & \\
& $5 \%$ level & -3.428581 & \\
& $10 \%$ level & -3.137711 & \\
\hline
\end{tabular}

Fuente: Elaborado con base a la serie de la producción de plátano.

Además de identificar que la serie es estacionaria, aparentemente estamos en presencia de una serie con quiebre estructural, lo cual verificamos con la prueba no paramétrica de punto de quiebre estructural de Chow para todo el periodo de la serie (Tabla 3). Por tanto, existe evidencia estadística para rechazar la hipótesis nula y concluimos que la serie si tiene un punto de quiebre estructural en el periodo 2017M08. 
Tabla 3. Prueba de punto de quiebre de Chow

\begin{tabular}{llll}
\hline F-statistic & 6.844015 & Prob. F(3,235) & 0.0002 \\
Log likelihood ratio & 20.18672 & Prob. Chi-Square(3) & 0.0002 \\
Wald Statistic & 50.78266 & Prob. Chi-Square(3) & 0.0000 \\
\hline
\end{tabular}

Fuente: Resultados obtenidos del programa Eviews.

Dada la presencia de quiebre estructural en la serie, utilizamos la prueba de Zivot Andrews para verificar si la serie con quiebre estructural es estacionaria o no. El valor de $t$-stadistic resultó $\tau=-4.990282$ y su $p-$ valor $=0.001720$, habiendo evidencia estadística rechazamos la hipótesis nula (con un nivel de significancia del $1 \%, 5 \%$ y $10 \%$ ) y se afirma que la serie con quiebre estructural no tiene raíz unitaria, por tanto, es estacionaria.

Para la estimación, se utilizaron gráficos de los valores de de autocorrelación simple y parcial, la FAS y FAP de la serie ajustada, de las gráficas concluimos que el mejor modelo para representar la producción del plátano es un ARIMA . En la Tabla 4, los valores estimados de sus parámetros son estadísticamente significativos ( $p$-valor $<0.01)$ de acuerdo a la probabilidad del $t$-stadistic. El nivel de significancia indica que las variables incorporadas en el modelo son los adecuados.

Tabla 4. Estimación del modelo ARIMA para la producción de plátano

\begin{tabular}{crrrr}
\hline \multirow{2}{*}{ Variable } & \multirow{2}{*}{ Coeficiente } & $\begin{array}{c}\text { Error } \\
\text { Estándar }\end{array}$ & t-Statistic & Prob. \\
\hline C & 22135.45 & 2240.785 & 9.878438 & 0.0000 \\
D1 & -6303.464 & 1557.728 & -4.046576 & 0.0001 \\
AR(1) & 0.953832 & 0.021639 & 44.07875 & 0.0000 \\
MA(1) & -0.223692 & 0.068635 & -3.259144 & 0.0013 \\
\hline
\end{tabular}

Fuente: Elaborado con base a la serie estacional de la producción de plátano

Para el diagnostico de los resultados, se realizó la prueba de normalidad de los residuos del modelo, la Figura 3(a) tiene la forma de una distribución normal, la prueba de Jarque-Bera resultó $J B=557.7$ con un $p$-valor $=0.000$, esto sucede por la existencia de un punto de quiebre estructural y por ende existe datos atípicos a consecuencia del punto de quiebre en la serie, lo que no se subsanaría la normalidad. Por otro lado, las raíces inversas del polinomio, son menores a uno y está dentro del circulo unitario (Figura 3b), lo que confirma que el modelo no se encuentra parametrizado y se puede usar para representar y pronosticar la producción del plátano.

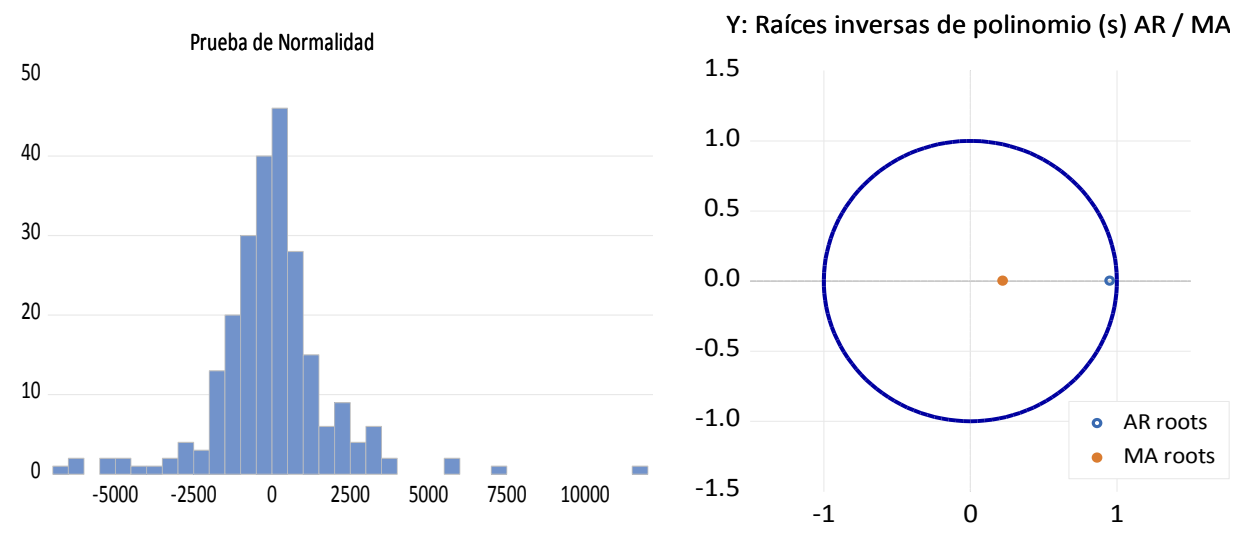

Fig. 3. Normalidad de los residuos y comprobación mediante raíces inversas del polinomio Fuente. Resultado obtenido con base a la serie en el programa Eviews 
B.Pronóstico para la producción del Plátano.

Luego de cumplir con el procedimiento de Box y Jenkins, se realizó el pronóstico de la producción del plátano para los próximos 24 periodos con el modelo ARIMA. La

Figura 4, muestra las predicciones del plátano con límites del 95\% de confianza, estos son los intervalos de confianza donde se ubica el verdadero valor en un tiempo futuro. Dado el mejor modelo ajustado y corroborado, los pronósticos presentan una tendencia decreciente para los próximos dos años.

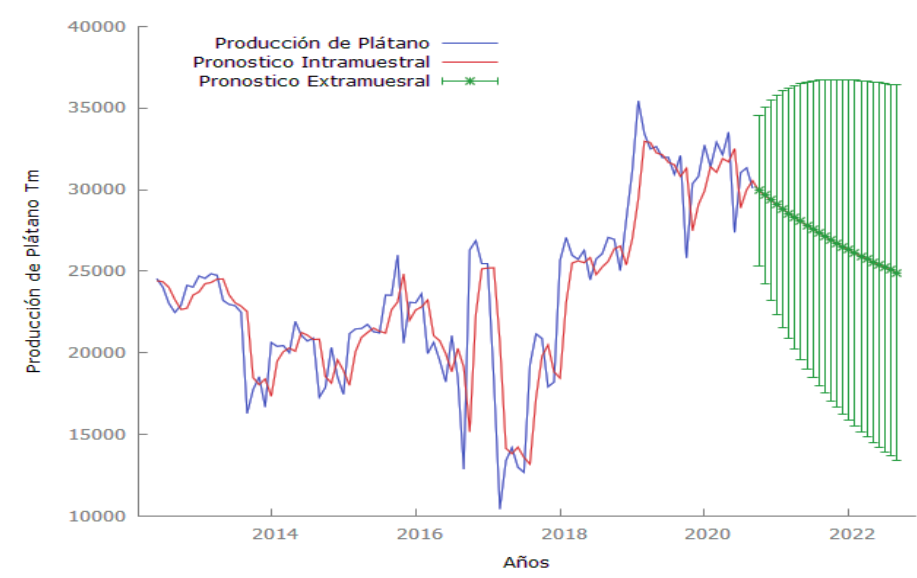

Fig. 4. Pronóstico de la producción de plátano periodo 2020M10 hasta 2022M09

Fuente: Serie de producción del plátano en el programa Eviews.

La producción del plátano, según el pronóstico para los siguientes 24 periodos, se ubica entre $29,851.12 \mathrm{Tm}$ para el periodo de 2020M10 y 24,803.57 Tm para el periodo 2022M09, tiene una tendencia decreciente ocasionados posiblemente por la coyuntura que estamos viviendo a nivel mundial (Tabla 5). Los criterios para validar los pronósticos son el MAPE (Mean Absolute Percent Error) resultando 18.59 porciento, que es un valor mínimo del error de predicción.

Tabla 5. Valores pronosticados para la producción del plátano en Tm

\begin{tabular}{|c|c|c|c|c|}
\hline Periodo & $\begin{array}{l}\text { Límite } \\
\text { inferior }\end{array}$ & Producción & $\begin{array}{c}\text { Límite } \\
\text { superior }\end{array}$ & Longitud \\
\hline $2020 M 10$ & 26008.82 & 29851.12 & 33693.43 & 7684.609 \\
\hline $2020 \mathrm{M} 11$ & 24693.98 & 29501.30 & 34308.62 & 9614.638 \\
\hline $2020 M 12$ & 23598.32 & 9167.44 & 4736.57 & 1138.25 \\
\hline 2021M01 & 22646.03 & 28848.82 & 35051.61 & 12405.57 \\
\hline 2021M02 & 21800.06 & 28544.74 & 35289.41 & 13489.35 \\
\hline 2021M03 & 21038.58 & 28254.53 & 35470.48 & 14431.90 \\
\hline 2021M04 & 20347.20 & 27977.57 & 35607.94 & 15260.74 \\
\hline 2021M05 & 19715.67 & 27713.25 & 35710.82 & 15995.15 \\
\hline 2021M06 & 19136.28 & 27460.99 & 35785.69 & 16649.41 \\
\hline 2021M07 & 1860 & 27220.24 & 35837.52 & 17234.55 \\
\hline 2021M08 & 18110.80 & 26990.48 & 35870.16 & 17759.36 \\
\hline 2021M09 & 17655.70 & 26771.20 & 35886.71 & 18231.02 \\
\hline $2021 \mathrm{M} 10$ & 17234.17 & 26561.94 & 35889.70 & 18655.53 \\
\hline $2021 \mathrm{M} 11$ & 16843.22 & 26362.22 & 35881.21 & 19037.99 \\
\hline $2021 \mathrm{M} 12$ & 16480.23 & 26171.61 & 35862.99 & 19382.76 \\
\hline $2022 \mathrm{M} 01$ & 16142.87 & 25989.71 & 35836.54 & 19693.67 \\
\hline 2022M02 & 15829.08 & 25816.10 & 35803.12 & 19974.03 \\
\hline 2022M03 & 15537.01 & 25650.42 & 35763.83 & 20226.81 \\
\hline 2022M04 & 15264.98 & 25492.30 & 35719.62 & 20454.63 \\
\hline 2022M05 & 15011.47 & 25341.39 & 35671.31 & 20659.84 \\
\hline 2022M06 & 14775.11 & 25197.37 & 35619.64 & 20844.53 \\
\hline 2022M07 & 14554.62 & 25059.93 & 35565.23 & 21010.61 \\
\hline 2022M08 & 14348.86 & 24928.75 & 35508.65 & 21159.79 \\
\hline 2022M09 & 14156.76 & 24803.57 & 35450.37 & 21293.61 \\
\hline
\end{tabular}




\section{Conclusiones}

De acuerdo a los resultados obtenidos, se concluye que el modelo autoregresivo integrado de medias móviles ARIMA(1,0,1) propuesto, fue el apropiado para representar la producción de plátano y para realizar el pronóstico para un periodo de 24 meses, desde 2020M10 hasta 2022M09 en la región de Piura: Cabe resaltar que la serie presentó una disminución en la producción de plátano en el periodo de 2017M03 posiblemente debido al fenómeno natural del niño, ocasionando un quiebre estructural en la serie.

Respecto a los pronósticos para los próximos dos años, la serie presenta una disminución en la producción de plátano en la región de Piura, resultando 29,851.12 Tm para el periodo 2020M10 y 24,803.57 Tm para el periodo 2022M09, con intervalos de confianza al $95 \%$ y sus longitudes correspondientes.

Este trabajo muestra la importancia de introducir los métodos de series de tiempo en el análisis de pronósticos productivos en el sector agrícola, con el objetivo de que los resultados puedan ser utilizados para la toma de decisiones de investigadores, productores de frutas y empresarios del sector agrícola en la región de Piura. Para trabajos futuros, se sugiere utilizar otros métodos de modelamiento y proyección para los cultivos de la región de Piura y cuyos resultados sirvan como base de comparación y promueva la investigación en temas agropecuarios.

\section{Referencias}

[1]A. A. S. Syed, A. Sajad, y U. J. Arshad, "Growth, Variability and Forecasting of Wheat and Sugarcane Production in Khyber Pakhtunkhwa, Pakistan," Agric. Res. Technol. Open Access J., 2018.

[2]Instituo Nacional de Estadistica e Informatica, "Producción Nacional - INEI," 2019.

[3]M. Laberry, "III Foro Nacional del Cultivo de Arroz," 2016.

[4]L. Torres, “Análisis Económico del Cambio Climático en la Agricultura de la Región Piura. Caso: Principales Productos Agroexportables," Consorc. Investig. Econ. y Soc. CIES, 2010.

[5]Instituto Nacional de Estadistica e Informatica, "Producto Bruto Interno Por Departamentos," 2019.

[6]D. Llico, "La minería, pesca y agricultura de Piura," monografias.com, 2013.

[7]H. Moyazzem, A. Faruq, y K. Ajit, "Forecasting of Banana Production in Bangladesh,” Am. J. Agric. Biol. Sci., 2016.

[8]J. Ruiz, G. Hernández, y R. Zulueta, “Análisis de series de tiempo en el pronóstico de la producción de caña de azúcar," Fac. Econ. - Univ. Veracruzana - Mex., 2010.

[9]V. Erossa, Proyectos de inversión en ingeniería: su metodología. 2004.

[10]A. Contreras, C. Atziry, M. José, y S. Diana, “Análisis de series de tiempo en el pronóstico de la demanda de almacenamiento de productos perecederos," Estud. Gerenciales 32 p.387-396 - Mex., 2016.

[11]G. Mendoza, "Pronosticar y métodos de pronóstico.,"
2003.

[12]A. Muñoz y F. Parra, Econometria aplicada, Ediciones. 2007.

[13]M. A. Hamjah, "Forecasting major fruit crops productions in Bangladesh using Box-Jenkins ARIMA model.," J. Econ. Sustain., vol. Dev., 5: 9, 2014.

[14]M. Casinillo y I. Manching, "Modeling the monthly production of banana using the box and Jenkins analysis.," Am. J. Agric. Biol. Sci., 2016.

[15]N. Suleman y S. Sarpong, "Forecasting Milled Rice Production in Ghana Using Box- Jenkins Approach," Int. J. Agric. Manag. Dev. (IJAMAD)., 2011.

[16]W. Merlin, "Modelo univariante de pronóstico del número de unidades de transfusión de sangre en el hospital regional Manuel Nuñez Butrón - Puno periodo 2006- 2015-I," Universidad Nacional del Altiplano - Puno, 2015.

[17]L. Laurente, "Proyección de la producción de papa en puno. una aplicación de la metodología de Box-Jenkins," Semest. Econ. - FIE - UNA Puno, 2018.

[18]Banco Central de Reserva del Perú, "Gerencia Central de Estudios Económicos," 2019. [Online]. Available: https:// estadisticas.bcrp.gob.pe/estadisticas/series/mensuales/resultados/PN01784AM/html.

[19]R. Hernández, C. Fernández, y M. del P. Baptista, Metodologia de la Investigación, vol. 6ta Ed. 2014.

[20]Banco Central de Reserva del Perú, "PIURA: Síntesis de Actividad Económica." 2020, [Online]. Available: https:// www.bcrp.gob.pe/estadisticas/informacion-regional/piura/ piura.html.

[21]I. Moumouni et al., "What happens between technico-institutional support and adoption of organic farming? A case study from Benin," Org. Agric., p. DOI 10.1007/s13165013-0039-x., 2013.

[22]U. Yule, "On a Method of Investigating Periodicities in Disturbed Series, with Special Reference to Wolfer's Sunspot Numbers," Philos. Trans. R. Soc. London, 1926.

[23]E. Slutsky, "The Summation of Random Causes as the Source of Cyclical Processes," Econom. 4 105-46, 1937. 1927.

[24]H. Wold, "A Study of the Analysis of Stationary Time Serie,” Uppsala: Almqvist and Wiksells., vol. 2nd ed.-19, 1938. [25]G. Box y G. M. Jenkins, "Time Series Analysis, Forecasting and Control," San Fr. Holden- Day, California, USA., 1976.

[26]D. Gujarati y D. Porter, Econometría. 2010.

[27]G. Box y D. Pierce, "Distribution of Residual Autocorrelations in Autoregressive Integrated Moving Average Time Series Models," J. Am. Stat. Assoc., vol. 65, p, 1970.

[28]G. Ljung y G. Box, "On a measure of lack of fit in time series models.," Biometrika, vol. V65: 297-3, 1978.

[29]C. Jarque y A. Bera, "A Test for Normality of Observations and Regression Residuals," Int. Stat. Inst., vol. Vol. 55, N, 1978.

[30]D. A. Dickey y W. A. Fuller, "Distribution of the Estimators for Autoregressive Time Series with a Unit Root," J. Am. Stat. Assoc., vol. 74, p, 1979. 
[31]P. C. B. Phillips y P. Perron, "Testing for a Unit Root in

1988.

Time Series Regression,” Biometrika, vol. 75, pp. 335-346,

\section{RESUMEN CURRICULAR}

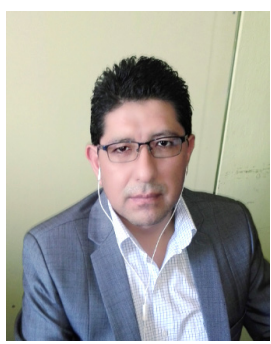

Freddy Carrasco Choque, Ingeniero Economista de la Universidad Nacional del Altiplano Puno y Magíster en Economía, Medio Ambiente y Recursos Naturales, en la Universidad de Los Andes, Bogotá Colombia. Docente ordinario asociado de la Universidad Nacional de Frontera.

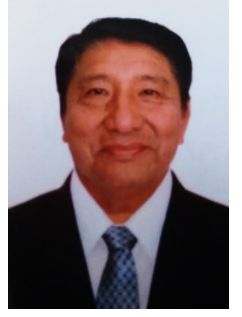

Mario Villegas Yarlequé, Economista con maestría en Ciencias de la Educación Superior. Investigador en la línea de Población y Desarrollo Sostenible. Docente ordinario auxiliar a tiempo completo en la Universidad Nacional de Frontera en la Facultad de Ingeniería Económica

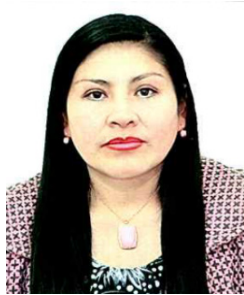

Janet Del Rocío Sánchez Castro, Ingeniero Economista y Magister en Admin istración y Finanzas de la Universidad Nacional del Altiplano, con Especialidad en Formulación, Evaluación de proyectos, especialización en Micro Finanzas, en Banca Financiera. 